\title{
Time-Delay Signatures in Multi-Transverse Mode VCSELs Subject to Double-Cavity Polarization-Rotated Optical Feedback
}

\author{
Hong Lin ${ }^{1}$, Aliza Khurram ${ }^{1,}$, and Yanhua Hong ${ }^{2}$ \\ 1. Department of Physics and Astronomy, Bates College, Lewiston, ME 04240, USA \\ 2. School of Electronic Engineering, Bangor University, Gwynedd, LL57 1UT, U.K. \\ Corresponding author's email address: hlin@bates.edu
}

\begin{abstract}
Time delay (TD) signatures are studied experimentally in orthogonal polarizations and in individual transverse modes respectively in a VCSEL operating with three transverse modes. Different types of concealment of the TD signatures are observed when the polarization of feedback is rotated through large angles. Effects of feedback strength and external cavity length on the TD signatures are investigated. Weak feedback leads to better concealment of the TD signatures in the dominant polarization. When the roundtrip time difference between the two external cavities is close to a half of the relaxation oscillation period, the TD signatures are minimized.
\end{abstract}

*Current address: Department of Mechanical Engineering, Massachusetts Institute of Technology, Cambridge, MA 02139 


\section{Introduction}

Optical chaos in semiconductor lasers has attracted a great deal of research interest [1]. It is shown that chaos can be used for secure communications [2-11], chaos radar [12] and lidar [13], random bit generation [14, 15], rainbow refractometry [16] and optical time-domain reflectometry $[17,18]$. Delayed optical feedback is a convenient method to induce chaos and other dynamical behaviors in semiconductor lasers [19-21], and is widely used for creating chaotic carrier in chaos-based secure communications [2-4, 7$11]$ and for generation of ultra-fast random bit sequences [14, 15]. In this scheme of chaos generation, an important parameter, the time delay (TD) signature due to the round-trip time in the external cavity, can be extracted from the power spectrum of the laser or the autocorrelation function (ACF) of the time series of the laser. Identification of the TD information makes it possible to reconstruct the chaotic system and hence compromise the security of chaotic encryption [22-24]. For ultra-fast random bit generation, the TD signature can seriously affect the statistical performance of random bit sequences $[14,15]$. Therefore suppression of TD signatures in chaotic semiconductor lasers has become an active research topic recently [25 -35].

Vertical-cavity surface-emitting lasers (VCSELs) are a special type of semiconductor lasers with a novel design for optical cavity. Compared to edge-emitting lasers, VCSELs have many unique features, such as low threshold current, large modulation bandwidth, single longitudinal mode operation, circular beam profile, and ease of fabrication of VCSEL arrays. These features make VCSEL very useful for opticfiber communications [36]. Correspondingly, concealment of TD signatures has been extensively studied in the single-transverse-mode regime of VCSELs [28-31, 33-35].

When the bias current increases, the output power of a VCSEL increases accompanied usually with the lasing of higher order transverse modes due to the spatial hole burning effect [37]. Given their low cost, low power consumption, and lower modal noise, multimode VCSELs have been the dominant component in short reach links and are good candidate for super-computing applications [38]. In addition, due to lack of polarization anisotropy, the emission of a VCSEL can be polarized along two orthogonal directions. Often, the light of a VCSEL is linearly polarized in a preferred direction. As 
the bias current or temperature changes, the polarization may switch to the orthogonal direction [39]. The polarization and transverse dynamics of VCSELs have been exploited in chaos-based secure communications. Synchronization between the master and slave lasers has been achieved in one of the two polarizations $[5-7,11]$ or in both polarizations [8]. Chaos synchronization between two multi-transverse-mode VCSELs shows that each transverse mode can be synchronized with its counterpart, implying the possibility of multi-channel chaos communications [9-11]. Therefore, it is important to investigate the TD features in multi-transverse mode VCSELs.

We have shown that TD signatures in a single transverse mode VCSEL subject to double-cavity, polarization-rotated optical feedback can been suppressed effectively in certain parameter regimes [34]. In this paper, we report the first experimental study on TD signatures in the multi-transverse-mode regime by applying double-cavity polarization-rotated optical feedback to the VCSEL. Our study focuses on the TD signatures in each polarization as well as in individual transverse modes. The results show that the TD signatures can be concealed at large angles of polarization. Interestingly, the TD signatures are concealed differently for different transverse modes.

\section{Polarization and Spectral Features of the VCSEL}

The experimental setup is shown in Fig. 1. The output of the VCSEL, collimated by the lens $\mathrm{L}$, is split at nonpolarizing beamsplitters $\mathrm{BS}_{1}$ and $\mathrm{BS}_{2}$, respectively. The VCSEL has two external cavities, formed by mirrors $\mathrm{M}_{1}$ and $\mathrm{M}_{2}$ respectively. The effective length of the first cavity (the optical path length between $\mathrm{M}_{1}$ and the VCSEL) is termed as $\mathrm{L}_{1}$, and the effective length of the second cavity is $\mathrm{L}_{2}$. The value of $\mathrm{L}_{2}$ is fixed at 56 $\mathrm{cm}$ whereas $\mathrm{L}_{1}$ is varied in our experiment. A neutral density filter, NDF, is inserted in cavity 1 to vary its reflectivity. The power reflectivity of cavity $1, R_{1}$, is given by $\left(\mathrm{R}_{B S 1} \mathrm{~T}_{N D F}\right)^{2} \mathrm{R}_{M}$, where RBS1 is the one-way reflectance of the beamsplitter $\mathrm{BS}_{1}$, $\mathrm{T}_{N D F}$ is the one-way transmittance of the neutral density filter, and RM1 is the power reflectivity of $\mathrm{M}_{1}$. The power reflectivity of cavity $2, \mathrm{R}_{2}$ is given by $\left(\mathrm{T}_{\mathrm{BS} 1} \mathrm{R}_{\mathrm{BS} 2}\right)^{2} \mathrm{R}_{\mathrm{M} 2}$, where $\mathrm{T}_{\mathrm{BS} 1}$ is the one-way transmittance of $\mathrm{BS}_{1}, \mathrm{R}_{\mathrm{BS} 2}$ is the one-way reflectance of $\mathrm{BS}_{2}$, and $\mathrm{R}_{\mathrm{M} 2}$ is the power reflectivity of $\mathrm{M}_{2}$. In our experiment, the value of $\mathrm{R}_{2}$ is fixed at $4.2 \%$. The angle through which the feedback polarization is rotated is named the angle of polarization, $\theta_{p}$. 
In our experiment, $\theta_{p}$ is adjusted with a quarter-wave plate (QWP) placed in each external cavity, and is set at the same value for both external cavities. The two orthogonal polarizations are separated at the polarizing beamsplitter (PBS) to enable simultaneous measurement of both polarizations. The polarized beams are sent to fiber couplers (FC), which allow us to record their time traces with fiber-coupled fast photodetectors (Newport 1580B, $12 \mathrm{GHz}$ ) and a digital oscilloscope (Tektronix TDS6804B, 8GHz). The sampling rate is $10 \mathrm{GS} / \mathrm{s}$ and the time duration is $10 \mu$ s for each time trace. The signal can also be sent to a Fabry-Perot (F-P) scanning spectrum analyzer (Exfo-Burleigh, FSR $750 \mathrm{GHz}$ ) for measuring the optical spectrum.

Fig. 2 gives the polarization-resolved power versus current curves of the VCSEL. As shown in Fig. 2a, the solitary VCSEL is linearly polarized from the threshold $\mathrm{I}_{\text {th }}$ to $\sim$ $3.15 \mathrm{~mA}$, with $\mathrm{I}_{\mathrm{th}}=2.69 \mathrm{~mA}$. This polarization is called the $\mathrm{X}$ polarization because it is parallel to the optical table. Polarization switching occurs at $3.18 \mathrm{~mA}$. This orthogonal polarization is perpendicular to the optical table and is thus termed the Y polarization. The VCSEL remains essentially Y-polarized until the bias current, $I$, reaches $\sim 4.0 \mathrm{~mA}$, after which the power of $\mathrm{X}$ polarization begins to rise. As the bias current is increased further, the powers of both the $\mathrm{X}$ and $\mathrm{Y}$ polarizations increase; however, the power of the $\mathrm{Y}$ polarizations is always greater than that of the $\mathrm{X}$ polarization. When the VCSEL is subject to double-cavity, polarization preserved (that is, $\theta_{p}=0^{\circ}$ ) optical feedback, the threshold current is reduced to $2.56 \mathrm{~mA}$, as shown in Fig. 2b. No polarization switching occurs. The $\mathrm{Y}$ polarization starts oscillation from $2.7 \mathrm{~mA}$ and surpasses the $\mathrm{X}$ polarization at around $2.9 \mathrm{~mA}$. For $I>3.2 \mathrm{~mA}$, the power of X polarization is higher than that of the solitary VCSEL, indicating that optical feedback can cause a transfer of energy from the $\mathrm{Y}$ polarization to the $\mathrm{X}$ polarization. The small fluctuations in the power of the two orthogonal polarizations, as shown in Fig. 2b, are anticorrelated, which is attributed to the polarization competition.

The solitary VCSEL operates in the fundamental mode regime for $I$ up to $4.1 \mathrm{~mA}$. Higher order transverse modes start oscillation when $I$ is above $4.1 \mathrm{~mA}$. In our experiment, the bias current is chosen to be $5.5 \mathrm{~mA}$, at which the VCSEL operates with three transverse modes: the fundamental mode (LP01 mode) and two first-order modes: 
$\mathrm{LP}_{11}{ }^{\mathrm{c}}$ and $\mathrm{LP}_{11}{ }^{\mathrm{s}}$ modes. The polarization-resolved optical spectra of the solitary VCSEL and the beam patterns of the transverse modes are given in [40]. The Y polarization consists of two strong modes, $\mathrm{LP} 01$ and $\mathrm{LP}_{11}{ }^{\mathrm{c}}$ modes, and a very weak $\mathrm{LP}_{11}{ }^{\mathrm{s}}$ mode. In the $\mathrm{X}$ polarization, the $\mathrm{LP}_{11} \mathrm{~s}$ mode - the one located in the middle of the spectrum-is the strongest mode and the $\mathrm{LP}_{01}$ mode is the weakest mode. The frequency of the $\mathrm{LP}_{11}{ }^{\mathrm{c}}$ mode is higher than that of the $\mathrm{LP}_{11}{ }^{\mathrm{s}}$ mode.

The power of each polarization changes when the polarization of optical feedback is rotated. Fig. 3a displays the polarized power as a function of the angle of polarization of optical feedback, $\theta_{p}$, for strong feedback $\left(\mathrm{R}_{1}=19.0 \%, \mathrm{R}_{2}=4.2 \%\right)$. As shown, the power of the $\mathrm{X}$ polarization increases notably for $\theta_{p}>50^{\circ}$. This is because that for a large value of $\theta_{p}$, a significant portion of the $\mathrm{Y}$ polarization is rotated to offer feedback to the $\mathrm{X}$ polarization. Since the power of the Y polarization is much higher than that of the $\mathrm{X}$ polarization, the power of the $\mathrm{X}$ polarization has a significant increase for large $\theta_{p}$. On the contrary, the variation in the Y-polarized power is small for large $\theta_{p}$ because a large portion of the feedback power comes from the $\mathrm{X}$ polarization, which is the weaker polarization of the VCSEL. When the feedback strength is weak $\left(\mathrm{R}_{1}=4.4 \%, \mathrm{R}_{2}=4.2 \%\right)$, the Y-polarized power is almost constant and the $\mathrm{X}$-polarized power only has a slight increase for large $\theta_{\mathrm{p}}$. This is expected since the mutual influence between the two polarizations is less for weaker feedback strength.

The optical spectra of the $\mathrm{Y}$ and $\mathrm{X}$ polarizations for two values of $\theta_{\mathrm{p}}-0^{\circ}$ and $90^{\circ}-$ are given in Fig. 4a and Fig. 4b, respectively. The strengths of feedback are the same as those used in Fig. 3a. The insets represent the optical spectra of the corresponding polarizations of the solitary VCSEL. Despite some changes in the peak intensities, the $\mathrm{LP}_{01}$ and $\mathrm{LP}_{11}{ }^{\mathrm{c}}$ mode remain the dominant modes in the $\mathrm{Y}$ polarization for both values of $\theta_{\mathrm{p}}$ (Fig. 4a). For the $\mathrm{X}$ polarization, polarization-preserved feedback $\left(\theta_{p}=0^{\circ}\right)$ enhances the $\mathrm{LP}_{01}$ mode and $\mathrm{LP}_{11}{ }^{\mathrm{c}}$ mode slightly. For $\theta_{p}$ of $90^{\circ}$, the spectral feature of the $\mathrm{X}$ polarization has a significant change. It manifests similarity to that of the $\mathrm{Y}$ polarization of the VCSEL: the intensity of the LP01 peak is the highest, and the intensity of the $\mathrm{LP}_{11}{ }^{\mathrm{c}}$ mode comparing to that of the $\mathrm{LP}_{01}$ mode resembles that in the $\mathrm{Y}$ polarization. This is 
because when $\theta_{p}$ is rotated to $90^{\circ}$, the power of the $\mathrm{Y}$ polarization is fed to the $\mathrm{X}$ polarization. The change is more obvious in the $\mathrm{X}$ polarized spectrum since the feedback power is from the strong $Y$ polarization. The changes in the optical spectra caused by $\theta_{p}$ are less obvious as the feedback strength is decreased, which agrees with the trend in the polarized power shown in Fig. 3.

When the polarization of optical feedback is rotated through a large angle, temporal fluctuation occurs in the optical spectrum of both polarizations. As an example, optical spectra of the X polarization at two different times are shown in Fig. 5 for $\theta_{p}$ of $70^{\circ}$, in which the variation in the $\mathrm{LP}_{01}$ mode is most obvious. The temporal fluctuation is also observed in the Y-polarized spectrum (not shown), but is smaller because a large portion of the feedback power comes from the weak X polarization. Note that the optical spectra do not fluctuate for small $\theta_{\mathrm{p}}$. Such time-dependent variation implies that large $\theta_{\mathrm{p}}$ can trigger competition among the transverse modes.

\section{Concealment of TD signatures}

\subsection{TD signatures in the $\mathrm{X}$ and $\mathrm{Y}$ polarizations}

Both polarizations manifest chaotic fluctuations when the VCSEL is subject to double-cavity, polarization rotated optical feedback. Generally speaking, the amplitude of fluctuations in the $\mathrm{X}$ polarization is greater than that in the $\mathrm{Y}$ polarization. This indicates that the $\mathrm{X}$ polarization is more sensitive to optical feedback.

The dynamics of the VCSEL is studied by recording the time series of the VCSEL at different angles of polarization, $\theta_{p}$. In our experiment, the value of $\theta_{p}$ is changed from $0^{\circ}$ to $90^{\circ}$ with an increment of $10^{\circ}$. Fig. 6 illustrates instability in the $\mathrm{X}$ polarization induced by double-cavity polarization-rotated optical feedback. The instability in the $\mathrm{Y}$ polarization is similar, which is not shown in the paper. In Fig. 6, the length of first cavity, $\mathrm{L}_{1}$, is $81 \mathrm{~cm}$. The corresponding time delay, $\tau_{1}\left(=2 \mathrm{~L}_{1} / \mathrm{c}\right)$, is $5.4 \mathrm{~ns}$. The power reflectivity of the first cavity, $\mathrm{R}_{1}$, is $19.0 \%$. The length of the second cavity, $\mathrm{L}_{2}$, is $56 \mathrm{~cm}$ and the corresponding time delay, $\tau_{2}\left(=2 \mathrm{~L}_{2} / \mathrm{c}\right)$, is $3.8 \mathrm{~ns}$. Its power reflectivity, $\mathrm{R}_{2}$, is $4.2 \%$. As shown in Fig. 6 , the time traces of the $\mathrm{X}$ polarization fluctuate irregularly with time. In the insets, the time series is plotted in a shorter time interval for the convenience 
of viewing the chaotic feature of the fluctuations. For each value of $\theta_{\mathrm{p}}$, the power spectrum is obtained by taking the Fourier transform of the time traces recorded over a $10 \mu \mathrm{s}$ time interval. For polarization preserved feedback $\left(\theta_{p}=0^{\circ}\right)$, as shown in Fig. 6(a2), the first few peaks are located at $0.19 \mathrm{GHz}\left(v_{1}\right), 0.34 \mathrm{GHz}\left(2 v_{2}-v_{1}\right), 0.54 \mathrm{GHz}$ $\left(2 v_{2}\right)$, and $0.73 \mathrm{GHz}\left(2 v_{2}-v_{1}\right)$, where $v_{1}=1 / \tau_{1}$ and $v_{2}=1 / \tau_{2}$ are resonance frequencies of cavity 1 and cavity 2 respectively. The TD signature of cavity 1 can be easily extracted from the power spectrum, whereas the TD signature of cavity 2 is less obvious. This may be due to the stronger feedback strength of the first cavity. When $\theta_{p}$ is $70^{\circ}$ (Fig. 6b2), the first few distinguishable peaks are located at $0.1 \mathrm{GHz}, 0.47 \mathrm{GHz}, 0.55 \mathrm{GHz}$, $0.65 \mathrm{GHz}$, and $0.74 \mathrm{GHz}$. However, none of them correspond to $\tau_{1}$ or $\tau_{2}$. For $\theta_{\mathrm{p}}$ of $80^{\circ}$ and $90^{\circ}$ (Fig. $6 \mathrm{c} 2$ and Fig. $6 \mathrm{~d} 2$ ), the first three peaks of $\sim-80 \mathrm{dBm}$ appear at $0.12 \mathrm{GHz}$, $0.54 \mathrm{GHz}$, and $0.64 \mathrm{GHz}$. Again, these peaks do not reveal any TD information. Therefore, the TD signatures are not visible in the power spectrum for large polarization angles.

Autocorrelation function (ACF) is a commonly used tool for identification of TD features [23-32]. The ACF is defined as

$$
C(\Delta t)=\frac{<[I(t+\Delta t)-<I(t+\Delta t)\rangle][I(t)-<I(t)\rangle]\rangle}{\sqrt{\left.\left.\left.\langle[I(t+\Delta t)-<I(t+\Delta t)\rangle]^{2}\right\rangle<[I(t)-<I(t)\rangle\right]^{2}\right\rangle}},
$$

where $I$ is the output intensity of the VCSEL, <.> denotes time average, and $\Delta \mathrm{t}$ is the delay time. We calculate the autocorrelation function by varying the delay time $\Delta \mathrm{t}$ from $0 \mathrm{~ns}$ to $30 \mathrm{~ns}$ at equal intervals of $0.1 \mathrm{~ns}$. It is known that the TD signatures measured from the ACF may not be located exactly at $\tau_{1}$ and $\tau_{2}[27,28]$. If the ACF peaks are located in the interval $\tau_{i}(1 \pm 5 \%)$, with $\mathrm{i}=1$ or 2 , then the TD information is considered retrievable. Fig. 7 gives the ACF of the $\mathrm{X}$ polarization as a function of $\Delta \mathrm{t}$ for different values of $\theta_{\mathrm{p}}$. When $\theta_{\mathrm{p}}$ is $0^{\circ}$, the highest peak in the ACF is located at $5.5 \mathrm{~ns}$, which corresponds to the TD in the first cavity, $\tau_{1}$. The other peaks are located at $3.8 \mathrm{~ns}\left(\tau_{2}\right)$, $1.7 \mathrm{~ns}\left(\tau_{1}-\tau_{2}\right), 7.2 \mathrm{~ns}\left(2 \tau_{1}-\tau_{2}\right), 9.3 \mathrm{~ns}\left(\tau_{1}+\tau_{2}\right), 11 \mathrm{~ns}\left(2 \tau_{1}\right)$, and other combinations of $\tau_{1}$ and $\tau_{2}$, as shown in Fig. 7a. The TD signatures can be identified easily from Fig. $7 \mathrm{a}$. When $\theta_{\mathrm{p}}$ is increased to $70^{\circ}$ (Fig. $7 \mathrm{~b}$ ), the value of the ACF displays a large fluctuation between -0.32 and 0.42 . The time interval between two adjacent peaks in the ACF is 0.6 
ns. Since the period of relaxation oscillation (RO) of the $\mathrm{X}$ polarization at this bias current is $0.88 \mathrm{~ns}$ [40], the time interval of $0.6 \mathrm{~ns}$ is close to $\tau_{1}-\tau_{2}-\tau_{\mathrm{RO}}$. The strongest peak is located at $1.7 \mathrm{~ns}$. The peaks representing TD characteristics are immersed in numerous surrounding peaks and are difficult to identify. The large variation in the ACF is also observed for $\theta_{\mathrm{p}}$ of $80^{\circ}$ (Fig. 7c) and $90^{\circ}$ (Fig. 7d). In Fig. 7c, the strongest peaks among a series of ACF peaks are located at $1.7 \mathrm{~ns}, 9.1 \mathrm{~ns}$, and $10.8 \mathrm{~ns}$, none of which corresponds to a TD signature. In Fig. 7d, the most distinguishable peaks in the ACF are located at $7.5 \mathrm{~ns}$ and $10.9 \mathrm{~ns}$. Again, the strongest ACF peaks do not appear at the characteristic delay times of the external cavities, $\tau_{1}$ and $\tau_{2}$, and therefore do not reveal any TD information. The ACF fluctuates with $\tau_{\mathrm{RO}}(0.9 \mathrm{~ns})$ in Fig. 7c and 7d. However, the peaks at $\tau_{1}$ and $\tau_{2}$ are slightly more negative than other peaks in Fig. $7 \mathrm{c}$ and $7 \mathrm{~d}$, so that their concealment is not as complete as in Fig. 7b. The negative value indicates anticorrelation after one round trip, which originates from the anticorrelated dynamics of the $\mathrm{X}$ and $\mathrm{Y}$ polarization [34]. In previous studies [25-34], TD signatures are concealed when the peaks corresponding to the time delays are suppressed. Fig. 7 gives a new type of concealment of the TD signatures, which we term as the "tree in forest" concealment based on the ACF feature.

Permutation entropy (PE) method [41] is also widely used to analyze time delay signatures [28-32]. In this method, the measured output intensity of a VCSEL has $\mathrm{N}$ samples $I_{t}$, where $t=1,2, \ldots N$. For a given time series $\{I t, t=1,2, \ldots, N\}$, let subsets $S_{q}$ contain $M$ samples $(M>1)$ of the measured intensities and define an embedding delay time $\tau=n T_{s}$, where $n$ is an integer and $T_{s}$ is the reciprocal of the sampling rate). The ordinal pattern of the subset is $\mathrm{S}_{\mathrm{q}}=[\mathrm{I}(\mathrm{t}), \mathrm{I}(\mathrm{t}+\tau), \ldots, \mathrm{I}(\mathrm{t}+(\mathrm{M}-1) \tau)]$. For practical purposes, $M$ is typically chosen between 3 and 7 [41]. In this paper, $M$ is set at $4 . \mathrm{S}_{\mathrm{q}}$ can be arranged as $\left[\mathrm{I}\left(\mathrm{t}+\left(\mathrm{r}_{1}-1\right) \tau \leq \mathrm{I}\left(\mathrm{t}+\left(\mathrm{r}_{2}-1\right) \tau \leq \ldots \leq \mathrm{I}\left(\mathrm{t}+\left(\mathrm{r}_{\mathrm{M}}-1\right) \tau\right)\right]\right.\right.$. Hence, any subset can be uniquely mapped into an "ordinal pattern" $\pi=\left(\mathrm{r}_{1}, \mathrm{r}_{2}, \ldots, \mathrm{r}_{\mathrm{M}}\right)$, which is one of the permutations of subset $\mathrm{S}_{\mathrm{q}}$ with $\mathrm{M}$ dimensions. For all the $\mathrm{M}$ ! possible permutations, the probability distribution $\mathrm{p}(\pi)$ is defined as [31]

$$
p(\pi)=\frac{\#\left\{t \mid t \leq N-M-n+1 ; S_{q} \text { has type } \pi\right\}}{N-M-n+1}
$$


where \# stands for "number." From the probability $\mathrm{p}(\pi)$, the PE is defined as

$$
h(p)=-\sum p(\pi) \log p(\pi) . \text { The normalized PE is given by } H_{P}=\frac{h(p)}{\log (M !)}[28
$$
30].

Fig. 8 gives the normalized $\mathrm{PE}$ of the $\mathrm{X}$ polarization for the same parameters as in Fig. 7. For $\theta_{\mathrm{p}}$ of $0^{\circ}$ (Fig. 8a), a large drop occurs at $5.5 \mathrm{~ns}\left(\tau_{1}\right)$, so that the TD signature of cavity 1 can be identified easily. Other smaller drops are located at $\tau_{2}$ and various combinations of the two delayed times, such as $3.8 \mathrm{~ns}\left(\tau_{2}\right), 1.7 \mathrm{~ns}\left(\tau_{1}-\tau_{2}\right), 9.3 \mathrm{~ns}\left(\tau_{1}+\tau_{2}\right)$, and $14.8 \mathrm{~ns}\left(2 \tau_{1}+\tau_{2}\right)$. When $\theta_{\mathrm{p}}=70^{\circ}$, the normalized PE demonstrates a lot of large troughs, as shown in Fig. 8b. The largest trough is located at $12.5 \mathrm{~ns}$, which does not represent either $\tau_{1}$ or $\tau_{2}$. The trough closest to $\tau_{1}$ is at $5.7 \mathrm{~ns}$. Its depth is similar to other troughs located at $1.7 \mathrm{~ns}, 6.8 \mathrm{~ns}, 18.2 \mathrm{~ns}, 23.9 \mathrm{~ns}$, and $25.1 \mathrm{~ns}$. The drop closest to $\tau_{2}$ is even smaller. So the TD signature of each external cavity is concealed just like a tree is concealed in a forest. As $\theta_{p}$ is increased further, the most distinguishable dips in $\mathrm{H}_{\mathrm{p}}$ occur at times not related to the TD signatures. For instance, the three deepest dips are at $1.7 \mathrm{~ns}\left(\tau_{1}-\tau_{2}\right), 7.5 \mathrm{~ns}\left(\sim 2 \tau_{1}-\tau_{2}\right)$, and $9.1 \mathrm{~ns}\left(\sim \tau_{1}+\tau_{2}\right)$ in Fig. 8c. In Fig. 8d, the most distinguishable dips are at $7.5 \mathrm{~ns}, 9.2 \mathrm{~ns}$, and $10.9\left(\sim 2 \tau_{1}\right.$. $)$ ns. The TD signatures cannot be retrieved from the PE function.

Similar features are also observed in the $\mathrm{Y}$ polarization. The ACF of the $\mathrm{Y}$ polarization is given in Fig. 9 for the same parameters as those for the $\mathrm{X}$ polarization in Fig.8. For polarization preserved feedback $\left(\theta_{\mathrm{p}}=0^{\circ}\right)$, the peak corresponding to $5.5 \mathrm{~ns}\left(\tau_{1}\right)$ can be easily identified (Fig. 9a). The other sharp peaks are at $3.8 \mathrm{~ns}\left(\tau_{2}\right), 1.7 \mathrm{~ns}\left(\tau_{1}-\tau_{2}\right)$, $9.3 \mathrm{~ns}\left(\tau_{1}+\tau_{2}\right), 11 \mathrm{~ns}\left(2 \tau_{1}\right)$, and $14.8\left(2 \tau_{1}+\tau_{2}\right)$. When $\theta_{\mathrm{p}}$ is increased to $70^{\circ}$, the characteristic peaks become immersed in numerous peaks of similar or larger amplitudes (Fig 9b). For $\theta_{\mathrm{p}}$ of $80^{\circ}$ (Fig. 9c), the ACF still consists of many peaks. The two peaks located at $\tau_{1}$ and $\tau_{2}$ may attract hackers' attention since they are more negative than others, similar to what is shown in Fig. 7c. As $\theta_{\mathrm{p}}$ is rotated to $90^{\circ}$, the ACF looks like a group of wave packets, with the most positive peaks at $1.6 \mathrm{~ns}\left(\tau_{1}-\tau_{2}\right), 7.5 \mathrm{~ns}\left(2 \tau_{2}\right), 9.3 \mathrm{~ns}$ 
$\left(\tau_{1}+\tau_{2}\right)$ and the most negative peaks at $2.1 \mathrm{~ns}, 7.2 \mathrm{~ns}$, etc. It is difficult to identify the TD signatures from Fig. 9d, too.

\subsection{TD signatures in each transverse mode}

We have measured the autocorrelation functions of three transverse modes: the fundamental mode and the $\mathrm{LP}_{11}{ }^{\mathrm{c}}$ mode in the $\mathrm{Y}$ polarization, and the $\mathrm{X}$-polarized $\mathrm{LP}_{11} \mathrm{~s}$ mode. As shown in Fig. 4, the first two modes are the strongest in the Y polarization; the $\mathrm{LP}_{11} \mathrm{~s}$ mode is the strongest in the $\mathrm{X}$ polarization for the solitary VCSEL and when the VCSEL is subject to polarization preserved optical feedback. Each transverse mode was selected with an etalon. The experimental parameters were the same as those for the $\mathrm{X}$ and Y polarizations (Figs. 7-9). Fig. 10 gives the ACF of the Y-polarized LP 01 mode. As shown in Fig. 10a, the two strongest peaks are located at $5.5 \mathrm{~ns}\left(\tau_{1}\right)$ and $3.8 \mathrm{~ns}\left(\tau_{2}\right)$, and the peaks corresponding to their combinations (e. g., $\tau_{1}-\tau_{2}, \tau_{1}+\tau_{2}, \ldots$ ) are weaker for polarization preserved feedback. Hence the TD signatures are easily recognized. In Fig. $10 \mathrm{~b}\left(\theta_{\mathrm{p}}=70^{\circ}\right)$, the ACF consists of numerous sharp peaks of similar amplitudes and the characteristic peaks representing TD signatures are hidden among them successfully. The peak standing out of the noisy background is located at $9.6 \mathrm{~ns}$, which corresponds neither $\tau_{1}$ nor $\tau_{2}$. Similarly, it is difficult to tell which peak represents time delay among a group of similar peaks in Figs. 10c and 10d. Given that the optical spectrum displays temporal fluctuation for large $\theta_{\mathrm{p}}$ (Fig. 5), the origin of multiple peaks in the ACF may be related to competition between the fundamental mode and other modes.

The autocorrelation function of the Y-polarized $\mathrm{LP}_{11}{ }^{\mathrm{c}}$ mode is given in Fig. 11. For $\theta_{\mathrm{p}}$ of $0^{\circ}$, two strongest peaks are located at $\tau_{1}$ and $\tau_{2}$ in Fig. 11a. The magnitudes of these two peaks are less than those in Fig. 10a because the $\mathrm{LP}_{11}{ }^{\mathrm{c}}$ mode is weaker than the fundamental mode. The peaks representing $\tau_{1}$ and $\tau_{2}$ decrease with increasing $\theta_{\mathrm{p}}$. When $\theta_{\mathrm{p}}$ is $70^{\circ}$ (Fig. 11b) and $80^{\circ}$ (Fig. 11c), the peak at $\tau_{2}$ becomes very weak. As $\theta_{\mathrm{p}}$ reaches $90^{\circ}$, the TD signature of cavity 2 vanishes. The TD of cavity 1 is still visible though it is decreased considerably. Hence, a partial concealment is achieved in the $\mathrm{LP}_{11}{ }^{\mathrm{c}}$ mode for large $\theta_{\mathrm{p}}$. This is different from the "tree in forest" concealment in the fundamental mode.

Fig. 12 is for the $\mathrm{X}$-polarized $\mathrm{LP}_{11}{ }^{\mathrm{s}}$ mode, the weakest among the three selected modes. Similar to the other two modes, the TD signatures can be easily identified from 
its ACF for polarization preserved feedback. For $\theta_{p}$ of $70^{\circ}$, the peak representing $\tau_{1}$ is barely visible and that for $\tau_{2}$ is greatly suppressed. When $\theta_{\mathrm{p}}$ is increased further, some periodicity occurs. The period of the ACF in Fig. $12 \mathrm{c}\left(\theta_{\mathrm{p}}=80^{\circ}\right)$ is approximately $\tau_{1}-\tau_{2}$, similar to that in Fig. 10c. This may indicate that the dynamics of the X-polarized $\mathrm{LP}_{11} \mathrm{~s}$ mode is somewhat influenced by the Y-polarized fundamental mode through polarization rotated feedback. For $\theta_{\mathrm{p}}$ of $90^{\circ}$, the period in ACF is $0.8 \mathrm{~ns}$, which is close to the period of relaxation oscillation of the mode. Comparing the ACFs of these transverse modes to that of each polarization, we can conclude that the fundamental mode is a dominant contributor to the multiple peak features in the ACF. In other words, the "tree in forest" type of concealment is mainly induced by the fundamental mode.

\subsection{Effect of feedback strength}

For weak optical feedback, the TD features in the $\mathrm{X}$ and $\mathrm{Y}$ polarizations manifest some differences, as shown in Fig. 13. For both polarizations, their ACFs reveal the TD signatures by the peaks located at $5.5 \mathrm{~ns}\left(\tau_{1}\right)$ and $3.8 \mathrm{~ns}\left(\tau_{2}\right)$ when $\theta_{\mathrm{p}}$ is $0^{\circ}$. As $\theta_{\mathrm{p}}$ is increased to $70^{\circ}$, the peaks corresponding to the TD signatures are somewhat decreased for the $\mathrm{X}$ polarization (Fig. 13b). For $\theta_{\mathrm{p}}$ of $80^{\circ}$, the TD signatures are weakened further in the X polarization (Fig. 13c). This trend of variation is qualitatively the same as that observed in the $\mathrm{LP}_{11}{ }^{\mathrm{c}}$ mode in Fig.11. Different from the case of strong feedback (Fig. 7), the ACF of the X polarization for weak feedback does not have multiple peaks of similar amplitudes for large $\theta_{\mathrm{p}}$. This indicates that the influence of the fundamental mode on the $\mathrm{X}$ polarization is less due to weaker feedback. On the other hand, the ACF of the Y polarization has numerous peaks of similar amplitudes for large $\theta_{p}$ (Figs. 13e and 13f),

successfully producing a "tree in forest" concealment of the TD signatures. This suggests that the dynamics of the Y polarization is dominated by the fundamental mode. By comparing Fig. $13 \mathrm{f}$ to Fig. 9c, we can see that the TD signatures in the Y polarization are concealed more thoroughly for weak feedback. This agrees with the results in some previous studies in the single transverse mode regime $[28,30]$.

\subsection{Effect of External Cavity Length}

Similar TD behaviors are obtained for other values of L1. Fig. 14 illustrates an 
example for $L_{1}$ of $36 \mathrm{~cm}$. The other parameters are the same as those in Fig. 13. The round-trip time in cavity $1, \tau_{1}$, is $2.4 \mathrm{~ns}$. For $\theta_{\mathrm{p}}$ of $0^{\circ}$, the ACF of both the $\mathrm{X}$ and $\mathrm{Y}$ polarizations manifest sharp peaks at $2.5 \mathrm{~ns}\left(\tau_{1}\right)$ and $3.8 \mathrm{~ns}\left(\tau_{2}\right)$, as given in Figs. 14a and $14 \mathrm{~d}$, respectively. When $\theta_{\mathrm{p}}$ is increased to $70^{\circ}$, the peaks representing $\tau_{1}$ and $\tau_{2}$ decrease but are still distinguishable for the $\mathrm{X}$ polarization (Fig. 14b). For $\theta_{\mathrm{p}}$ of $80^{\circ}$, the multipeak feature appears in the ACF of the $\mathrm{X}$ polarization (Fig. 14c). The peak corresponding to $\tau_{2}$ is concealed among numerous peaks, but the peak at $\tau_{1}$ stands out among other peaks. Thus partial concealment is obtained for the $\mathrm{X}$ polarization. The multi-peak feature in Fig. 14c implies a greater influence of the fundamental mode on the $\mathrm{X}$ polarization, which may be related to the feedback strength. Though the cavity reflectivity $R_{1}$ is the same in both Fig. 13 and Fig. 14, the cavity length is much longer in the former case. A slight imperfection in beam alignment can result in diffraction loss, and the loss increases with the distance of propagation. Therefore, the actual feedback strength in a short cavity $\left(\mathrm{L}_{1}=36 \mathrm{~cm}\right)$ may be higher than that in a long cavity $\left(\mathrm{L}_{1}=81\right.$ $\mathrm{cm})$, which increases the contribution of the fundamental mode to the $\mathrm{X}$ polarization in Fig. 14c. For the Y polarization, multiple peaks appear for both $70^{\circ}$ (Fig. 14e) and $80^{\circ}$ (Fig. 14f), and the characteristic peaks representing TD signatures are concealed successfully among them.

It has been shown numerically that the relaxation oscillation period, $\tau_{\mathrm{RO}}$, plays a role in concealing the TD signatures (for example, $[25,26,29]$ ). When the time delay in the single external cavity setup is close to $\tau_{R O}$, the TD signature is concealed $[25,26]$. For the double external cavity setup, the TD signatures are minimized when the difference between the round-trip times in the two external cavities, $\left|\tau_{1}-\tau_{2}\right|$, is close to $\tau_{\mathrm{RO}} / 2$ [29]. Fig. 15 gives the values of the ACF peaks located at $\tau_{1}$ and $\tau_{2}$, named $\mathrm{C}_{1}$ and $\mathrm{C}_{2}$ respectively, as a function of $\mathrm{L}_{1}$ for the $\mathrm{X}$ polarization at $5.5 \mathrm{~mA}$. The value of $\mathrm{L}_{2}$ is fixed at $56 \mathrm{~cm}$. Note that the angle of polarization is $60^{\circ}$, at which the TD signatures are decreased but not completely suppressed. Given that $\tau_{\mathrm{RO}}$ of the $\mathrm{X}$ polarization is $0.88 \mathrm{~ns}$ [40], the calculated values of $L_{1}$ for achieving $\left|\tau_{1}-\tau_{2}\right|=\tau_{\mathrm{RO}} / 2$ are $49.5 \mathrm{~cm}$ and $62.5 \mathrm{~cm}$, respectively. In Fig. 15, both $\mathrm{C}_{1}$ and $\mathrm{C}_{2}$ reach the highest value at $56 \mathrm{~cm}$; that is when $\mathrm{L}_{1}$ equals L2. This is similar to what was observed in a single-transverse-mode VCSEL [34] 
and may be caused by the resonance of two external cavities. $\mathrm{C}_{1}$ has two local minima, located at $46 \mathrm{~cm}$ and $66 \mathrm{~cm}$ respectively. The two local minima of $\mathrm{C}_{2}$ are at $49.5 \mathrm{~cm}$ and $66 \mathrm{~cm}$. This is qualitatively similar to the result in [29]. However, most locations of the local minima are a few centimeters off the calculated values. This discrepancy may be attributed to the multimode operation of the $\mathrm{X}$ polarization whereas the numerical result in [29] is for single-transverse mode operation. The values of $C_{1}$ and $C_{2}$ are the lowest for the longest $L_{1}$, which may be attributed to the lower actual feedback strength caused by the diffraction loss when light travels a longer distance.

\section{Conclusion}

Our results show that the TD signatures can be concealed differently in different transverse modes. In the fundamental mode, a type of "tree in forest" concealment is achieved when optical feedback is rotated through large angles. This is a new type of TD concealment, to which the competition among the transverse modes may contribute. In the $\mathrm{LP}_{11}{ }^{\mathrm{c}}$ mode and $\mathrm{LP}_{11}^{\mathrm{s}}$ mode, the TD signature of one external cavity is eliminated while the other is greatly suppressed. The modal behaviors affect collective concealment in both polarizations. When the fundamental mode plays the dominant role in dynamics, the "tree in forest" concealment is observed in both $\mathrm{X}$ and Y polarizations. Otherwise, the "tree in forest" concealment is observed in the $\mathrm{Y}$ polarization in which the fundamental mode is the strongest, whereas the TD signature of the second cavity is concealed in the $\mathrm{X}$ polarization. The effects of feedback strength, external cavity length, and relaxation oscillation on the TD signatures are studied as well. It is shown that weaker feedback gives better concealment of the TD signatures in the Y polarization, which agrees with the results in $[28,30]$. For the same feedback strength, the dynamical feature of the fundamental mode is weaker for a longer external cavity. This may be related to the increasing diffraction loss with longer cavity length. The TD signatures are weakened when the round-trip time difference between the two external cavities, $\left|\tau_{1}-\tau_{2}\right|$, is close to a half of the relaxation oscillation period, $\tau_{\mathrm{RO}}$. This is qualitatively similar to the theoretical result in a single transverse mode VCSEL [29]. 


\section{Acknowledgement}

This work was supported by the National Science Foundation, USA, under the Grant No. PHY-1068789. 


\section{References:}

[1] M. Sciamanna and K. A. Shore, Physics and applications of laser diode chaos, Nature Photon. 9 (2015) 151-162.

[2] C. Masoller, Anticipation in the synchronization of chaotic semiconductor lasers with optical feedback, Phys. Rev. Lett. 86 (2001) 2782-2785.

[3] D. W. Sukow, K. L. Blackburn, A. R. Spain, K. J. Babcock, J. V. Bennett and A. Gavrielides, Experimental synchronization of chaos in diode lasers with polarization-rotated feedback and injection, Opt. Lett. 29 (2004) 2393-2395.

[4] A. Argyris, D. Syvridis, L. Larger, V. Annovazzi-Lodi, P. Colet, I. Fischer, J. Garcia-Ojalvo, C. R.Mirasso, L. Pesquera, and K. A. Shore, Chaos-based communications at high bit rates using commercial fibre-optic links, Nature, 438 (2005) 343-346.

[5] N. Fujiwara, Y. Takiguchi, and J. Ohtsubo, Observation of the synchronization of chaos in mutually injected vertical-cavity surface-emitting semiconductor lasers, Opt. Lett. 28 (2003) 1677-1679.

[6] A. Quirce, A. Valle, H. Thienpont, and K. Panajotov, Chaos synchronization in mutually coupled $1550-\mathrm{nm}$ vertical-cavity surface-emitting lasers with parallel polarizations and long delay time, J. Opt. Soc. Am. B 33 (2016) 90-98.

[7] M. W. Lee, Y. Hong, and K. A. Shore, Experimental demonstration of VCSELbased chaotic optical communications, IEEE Photonics Technol. Lett. 16 (2004) 2392-2394.

[8] Y. Hong, M. W. Lee, P. S. Spencer, and K. A. Shore, Enhanced chaos synchronization in unidirectionally coupled vertical-cavity surface-emitting lasers with polarization-preserved injection, Opt. Lett. 33 (2008) 587-589.

[9] M. S. Torre, C. Masoller, K.A. Shore, Synchronization of unidirectionally injected multi-transverse-mode vertical-cavity surface-emitting lasers, J. Opt. Soc. Am. B 21 (2004) 1772-1780.

[10] X. Li, W. Pan, D. Ma, B. Luo, Chaos synchronization of unidirectionally coupled vertical-cavity surface-emitting lasers with global and mode selective coupling, Opt. Express 14 (2006) 3138-3151. 
[11] H. Lin, M. M. Valles, and Y. Zhang, Synchronization of chaotic outputs in multitransverse-mode vertical-cavity surface-emitting lasers, Opt. Commun. 309 (2013) 242-246.

[12] F.-Y. Lin and J.-M. Liu, Chaotic radar using nonlinear dynamics, IEEE J. Quantum Electron. 40 (2004) 815-820.

[13] F.-Y. Lin and J.-M. Liu, Chaotic lidar, IEEE J. Sel. Top. Quantum Electron. 10 (2004) 991-997.

[14] A. Uchida, K. Amano, M. Inoue, K. Kirano, S. Naito, H. Someya, I. Oowada, T. Kurashige, J. Shiki, S. Yoshimori, K. Yoshimura, and P. Davis, Fast physics random bit generation with chaotic semiconductor lasers, Nature Photon. 2 (2008) 728-732.

[15] I. Reidler, Y. Aviad, M. Rosenbluh, and K. Kanter, Ultrahigh-speed random number generation based on a chaotic semiconductor laser, Phys. Rev. Lett. 103 (2009) 024102.

[16] M. Peil, I. Fischer, W. Elsäßer, S. Bakic, N. Damascke, C. Tropea, S. Stry, and J. Sacher, Rainbow refractomery with a tailored incoherent semiconductor laser source, Appl. Phys. Lett. 89 (2006) 091106.

[17] A. Wang, N. Wang, Y. Yang, B. Wang, M. Zhang, and Y. Wang, Precise Fault Location in WDM-PON by Utilizing Wavelength Tunable Chaotic Laser, J. Lightwave Technol. 30 (2012) 3420-3426.

[18] L. Xia, D. Huang, J. Xu, and D. Liu, Simultaneous and precise fault locating in WDM-PON by the generation of optical wideband chaos, Opt. Lett. 38 (2013) 37623764.

[19] G. C. Dente, P. S. Durkin, K. A. Wilson, and C. E. Moeller, Chaos in the coherence collapse of semiconductor lasers, J. Quantum Electron. 24 (1988) 24412447.

[20] T. Sano, Antimode dynamics and chaotic itinerancy in the coherence collapse of semiconductor lasers with optical feedback, Phys. Rev. A 50 (1994) 2719-2726.

[21] P. S. Spencer, C. R. Mirasso, and K. A. Shore, Effect of strong optical feedback on vertical-cavity surface-emitting lasers, Photon. Technol. Lett. 10 (1998) 191-193.

[22] R. Hegger, M. J. Bünner, H. Kantz, and A. Giaquinta, Identifying and modeling delay feedback systems, Phys. Rev. Lett. 81 (1998) 558-561. 
[23] C. Zhou and C. H. Lai, Extracting messages masked by chaotic signals of timedelay systems, Phys. Rev. E 60 (1999) 320-323.

[24] M. D. Prokhorov, V. I. Ponomarenko, A. S. Karavaev, and B. P. Bezruchko, Reconstruction of time-delayed feedback systems from time series, Physica D 203 (2005) 209-223.

[25] D. Rontani, A. Locquet, M. Sciamanna, and D. S. Citrin, Loss of time-delay signature in the chaotic semiconductor laser with optical feedback, Opt. Lett. 32 (2007) 2960-2962.

[26] R. M. Nguimdo, G. Verschaffelt, J. Danckaert, and G. Van der Sande, Loss of time-delay signature in chaotic semiconductor ring lasers, Opt. Lett. 37 (2012) 25412543.

[27] J. G. Wu, G. Q. Xia, and Z. M. Wu, Suppression of time delay signatures of chaotic output in a semiconductor laser with double optical feedback, Opt. Express 17 (2009) 20124-20133.

[28] S. Xiang, W. Pan, B. Luo, L. Yan, X. Zou, N. Jiang, L. Yang, and H. Zhu, Conceal time-delay signature of chaotic vertical-cavity surface-emitting lasers by variable-polarization optical feedback, Opt. Commun. 284 (2011) 5758-5765.

[29] P. Xiao, Z.-M. Wu, J.-G. Wu, L. Jiang, T. Deng, X. Tang, L. Fan, and G.-Q. Xia, Time-delay signature concealment of chaotic output in a vertical-cavity surfaceemitting laser with double variable-polarization optical feedback, Opt. Commun. 286 (2013) 339-343.

[30] S. Priyadarshi, Y. Hong, I. Pierce, and K. A. Shore, Experimental investigation of time-delay signature concealment in chaotic external cavity VCSELs subject to variable optical polarization angle of feedback, IEEE J. Sel. Topics Quantum Electron. 19 (2013) 1700707.

[31] Y. Hong, Experimental study of time-delay signature of chaos in mutually coupled vertical-cavity surface-emitting lasers subject to polarization rotated optical feedback, Opt. Express 21 (2013) 17894-17903.

[32] S. Y. Xiang, W. Pan, A. J. Wen, N. Q. Li, L. Y. Zhang, L. Shang, and H. X. Zhang, Conceal time delay signature of chaos in semiconductor lasers with dual-path injection, Photon. Technol. Lett. 25 (2013) 1398-1401. 
[33] Z.-Q. Zhong, Z.-M. Wu, J.-G. Wu, and G.-Q. Xia, Time-delay signature suppression of polarization resolved chaos outputs from two mutually coupled VCSELs, IEEE Photon. J. 5 (2013) 1500409.

[34] H. Lin, Y. Hong, and K. A. Shore, Experimental study of time-delay signature in vertical-cavity surface-emitting lasers subject to double-cavity polarization-rotated optical feedback, J. Lightwave Technol. 32 (2014) 1829-1836.

[35] C.-H. Cheng, Y.-C. Chen, and F.-Y. Lin, Chaos time delay signature suppression and bandwidth enhancement by electrical heterodyning, Opt. Express 23 (2015) 2308-2319.

[36] K. Schires, A. Hurtado, I. D. Henning, and M. J. Adams, Polarization and timeresolved dynamics of a 1550-nm VCSEL subject to orthogonally polarized optical injection, IEEE Photon. J. 3 (2011) 555-563.

[37] A. Valle, J. Sarma, and K. A. Shore, Spatial holeburning effects on the dynamics of vertical cavity surface-emitting laser diodes, IEEE J. Quantum Electron. 31 (1995) 1423-1431.

[38] D. Mahgerefteh, C. Thompson, G. Denoyer, T. Nguyen, I. Lyubomirsky, C. Kocot, and J. Tatum, Techno-economic comparison of silicon photonics and multimode VCSELs, J. Lightwave Technol. 34 (2016) 233-242.

[39] K. D. Choquett, R.P. Schneider, K.L. Lear, and R.E. Leibenguth, Gain dependent polarization properties in vertical cavity lasers, J. Sel. Top. Quantum Electron. 1 (1995) 661-666.

[40] H. Lin, A. Khurram, M. Black-Ingersoll, and A. Valle, Polarization and modal dynamics of multimode vertical-cavity surface-emitting lasers subject to optical feedback and current modulation, Opt. Commun. 350 (2015) 178-188.

[41] C. Bandt and B. Pompe, Permutation entropy: A natural complexity measure for time series, Phys. Rev. Lett. 88 (2002) 174102. 


\section{Captions:}

Fig. 1. Experimental setup, where BS stands for nonpolarizing beamsplitter, FC is the fiber coupler, $\mathrm{L}$ is the collimating lens, $\mathrm{M}$ is the feedback mirror, $\mathrm{PD}$ is the photodetector, PBS is the polarizing beamsplitter, and QWP is the quarter-wave plate.

Fig. 2. Polarization resolved power versus current curves of (a) the solitary VCSEL, and (b) the VCSEL subject to polarization-preserved $\left(\theta_{p}=0^{\circ}\right)$, double-cavity feedback. The reflectivities of the external cavities 1 and 2 are $R_{1}=19.0 \%$ and $R_{2}=4.2 \%$ respectively. The cavity lengths are $\mathrm{L}_{1}=81 \mathrm{~cm}$ and $\mathrm{L}_{2}=56 \mathrm{~cm}$. (X: dashed; $\mathrm{Y}$ : solid).

Fig. 3. Polarization resolved output power versus $\theta_{p}$, the angle of polarization of feedback (square: $\mathrm{X}$; circle: Y). (a) $\mathrm{R}_{1}=19.0 \%$, (b) $\mathrm{R}_{1}=4.4 \%$. The other parameters are $\mathrm{I}=5.5 \mathrm{~mA}, \mathrm{R}_{2}=4.2 \%, \mathrm{~L}_{1}=81 \mathrm{~cm}$, and $\mathrm{L}_{2}=56 \mathrm{~cm}$.

Fig. 4. Optical spectra of (a) the $\mathrm{Y}$ polarization and (b) the $\mathrm{X}$ polarization for $\theta_{p}=0^{\circ}$ (solid curve) and $\theta_{p}=90^{\circ}$ (dashed curve) respectively. The other parameters are $\mathrm{I}=5.5 \mathrm{~mA}$, $\mathrm{R}_{1}=19.0 \%, \mathrm{R}_{2}=4.2 \%, \mathrm{~L}_{1}=81 \mathrm{~cm}$, and $\mathrm{L}_{2}=56 \mathrm{~cm}$. The insets represent the optical spectra of the solitary VCSEL.

Fig. 5. Optical spectrum of the $X$ polarization at two different times for $p$ of $70^{\circ}$. The other parameters are $\mathrm{I}=5.5 \mathrm{~mA}, \mathrm{R}_{1}=19.0 \%, \mathrm{R}_{2}=4.2 \%, \mathrm{~L}_{1}=81 \mathrm{~cm}$, and $\mathrm{L}_{2}=56 \mathrm{~cm}$.

Fig. 6. Time traces (left column) and their Fourier transforms (right column) of the $\mathrm{X}$ polarization for $\theta_{p}$ of (a) $0^{\circ}$, (b) $70^{\circ}$, (c) $80^{\circ}$, and (d) $90^{\circ}$ respectively. The other parameters are $\mathrm{I}=5.5 \mathrm{~mA}, \mathrm{R}_{1}=19.0 \%, \mathrm{R}_{2}=4.2 \%, \mathrm{~L}_{1}=81 \mathrm{~cm}$, and $\mathrm{L}_{2}=56 \mathrm{~cm}$. Insets are the time series in a shorter time interval.

Fig. 7. Autocorrelation function of the $\mathrm{X}$ polarization for $\theta_{p}$ of (a) $0^{\circ}$, (b) $70^{\circ}$, (c) $80^{\circ}$, and (d) $90^{\circ}$. The other parameters are $\mathrm{I}=5.5 \mathrm{~mA}, \mathrm{R}_{1}=19.0 \%, \mathrm{R}_{2}=4.2 \%, \mathrm{~L}_{1}=81 \mathrm{~cm}$, and $\mathrm{L}_{2}=56 \mathrm{~cm}$.

Fig. 8. Permutation entropy of the $\mathrm{X}$ polarization for $\theta_{p}$ of (a) $0^{\circ}$, (b) $70^{\circ}$, (c) $80^{\circ}$, and (d) $90^{\circ}$. The other parameters are $\mathrm{I}=5.5 \mathrm{~mA}, \mathrm{R}_{1}=19.0 \%, \mathrm{R}_{2}=4.2 \%, \mathrm{~L}_{1}=81 \mathrm{~cm}$, and $\mathrm{L}_{2}=56$ 
$\mathrm{cm}$.

Fig. 9. Autocorrelation function of the Y polarization for $\theta_{p}$ of (a) $0^{\circ}$, (b) $70^{\circ}$, (c) $80^{\circ}$, and (d) $90^{\circ}$. The other parameters are $\mathrm{I}=5.5 \mathrm{~mA}, \mathrm{R}_{1}=19.0 \%, \mathrm{R}_{2}=4.2 \%, \mathrm{~L}_{1}=81 \mathrm{~cm}$, and $\mathrm{L}_{2}$ $=56 \mathrm{~cm}$.

Fig. 10. Autocorrelation function of the Y-polarized fundamental mode for $\theta_{p}$ of (a) $0^{\circ}$, (b) $70^{\circ}$, (c) $80^{\circ}$, and (d) $90^{\circ}$. The other parameters are $\mathrm{I}=5.5 \mathrm{~mA}, \mathrm{R}_{1}=19.0 \%, \mathrm{R}_{2}=4.2 \%, \mathrm{~L}_{1}$ $=81 \mathrm{~cm}$, and $\mathrm{L}_{2}=56 \mathrm{~cm}$.

Fig. 11. Autocorrelation function of the Y-polarized $\mathrm{LP}_{11}{ }^{\mathrm{c}}$ mode for $\theta_{p}$ of (a) $0^{\circ}$, (b) $70^{\circ}$, (c) $80^{\circ}$, and (d) $90^{\circ}$. The other parameters are $\mathrm{I}=5.5 \mathrm{~mA}, \mathrm{R}_{1}=19.0 \%, \mathrm{R}_{2}=4.2 \%, \mathrm{~L}_{1}=81 \mathrm{~cm}$, and $\mathrm{L}_{2}=56 \mathrm{~cm}$.

Fig. 12. Autocorrelation function of the X-polarized $\mathrm{LP}_{11}{ }^{\mathrm{s}}$ mode for $\theta_{p}$ of (a) $0^{\circ}$, (b) $70^{\circ}$, (c) $80^{\circ}$, and (d) $90^{\circ}$. The other parameters are $\mathrm{I}=5.5 \mathrm{~mA}, \mathrm{R}_{1}=19.0 \%, \mathrm{R}_{2}=4.2 \%, \mathrm{~L}_{1}=81 \mathrm{~cm}$, and $\mathrm{L}_{2}=56 \mathrm{~cm}$.

Fig. 13. Autocorrelation functions for different values of $\theta_{\mathrm{p}}$. Upper row: the $X$ polarization; lower row: the $\mathrm{Y}$ polarization. The other parameters are $\mathrm{I}=5.5 \mathrm{~mA}$, $\mathrm{R}_{1}=4.4 \%, \mathrm{R}_{2}=4.2 \%, \mathrm{~L}_{1}=81 \mathrm{~cm}$, and $\mathrm{L}_{2}=56 \mathrm{~cm}$.

Fig. 14. Autocorrelation functions for different values of $\theta_{\mathrm{p}}$. Upper row: the $\mathrm{X}$ polarization; lower row: the $\mathrm{Y}$ polarization. The other parameters are $\mathrm{I}=5.5 \mathrm{~mA}, \mathrm{R}_{1}=4.4$ $\%, \mathrm{R}_{2}=4.2 \%, \mathrm{~L}_{1}=36 \mathrm{~cm}$, and $\mathrm{L}_{2}=56 \mathrm{~cm}$.

Fig. 15. The ACF peaks representing the TD signatures versus the length of the first external cavity. The experimental parameters are $\mathrm{I}=5.5 \mathrm{~mA}, \mathrm{R}_{1}=4.4 \%, \mathrm{R}_{2}=4.2 \%, \mathrm{~L}_{2}=56$ $\mathrm{cm}$, and $\theta_{\mathrm{p}}=60^{\circ}$. 


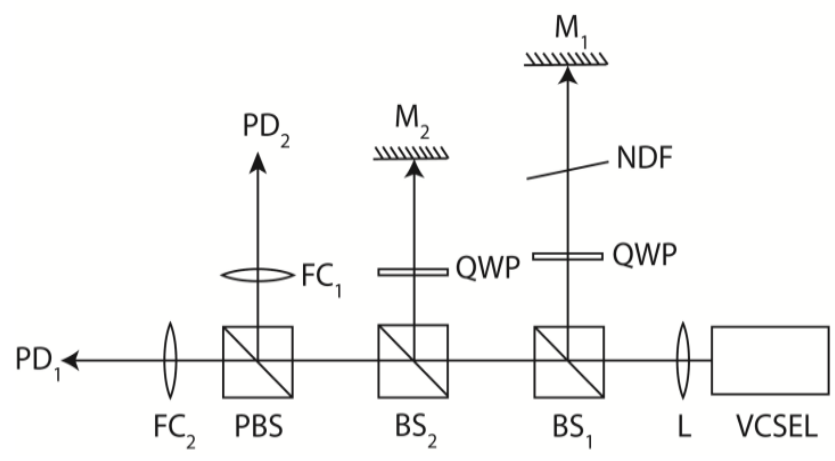

Fig. 1 

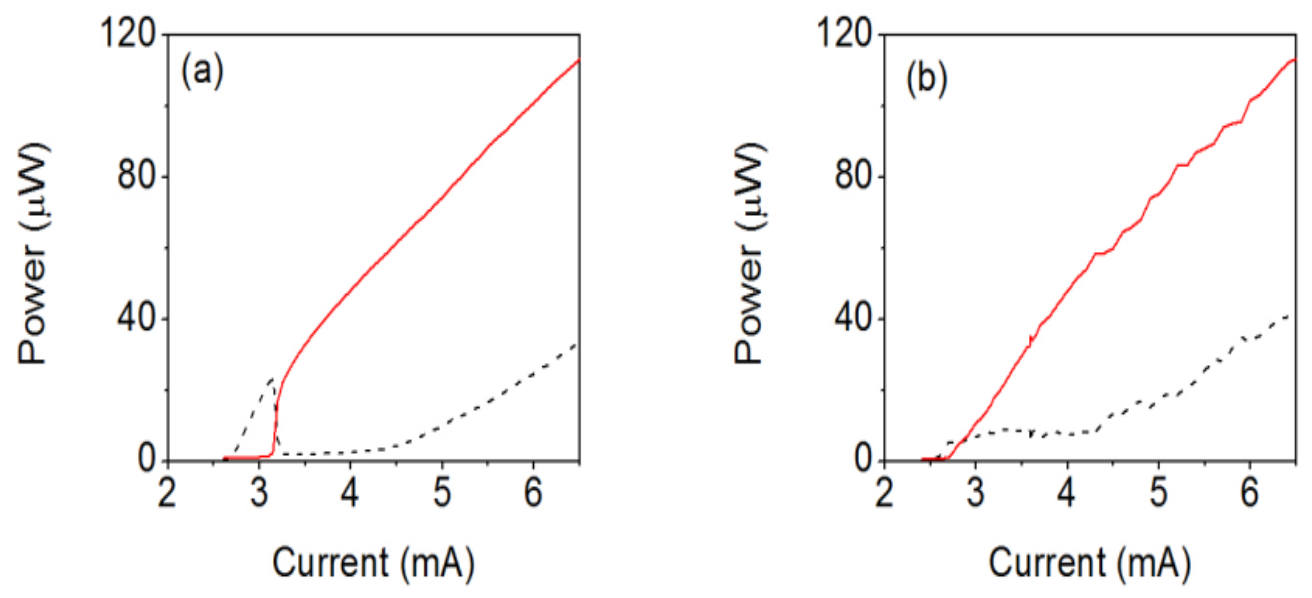

Fig. 2 

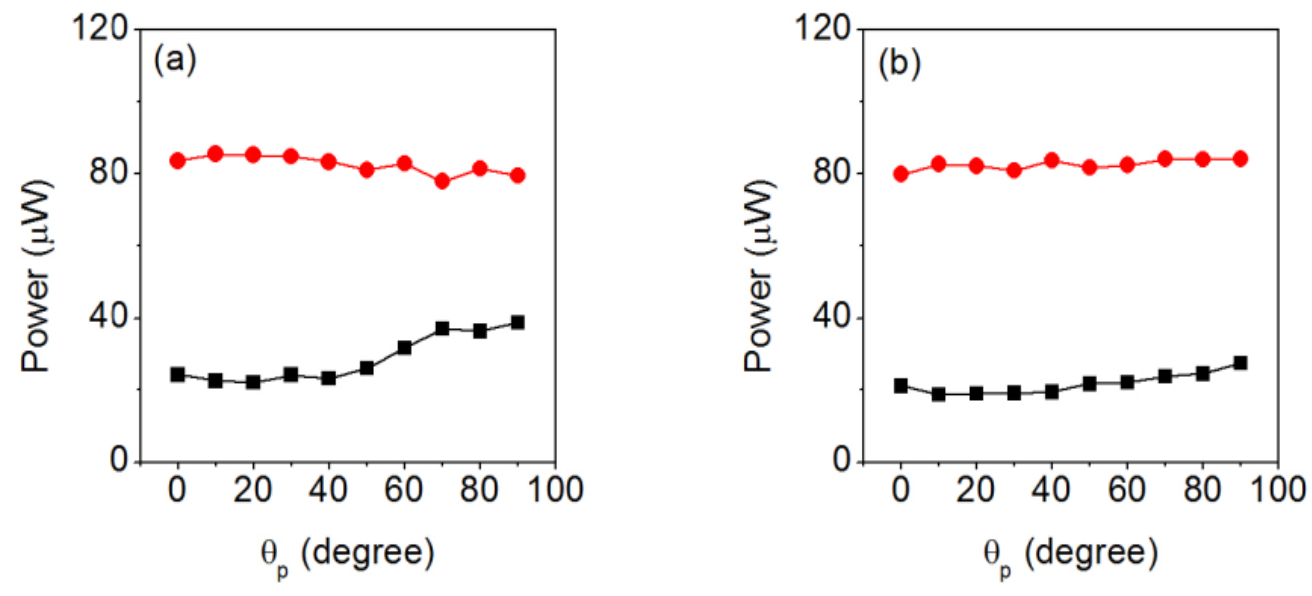

Fig. 3 


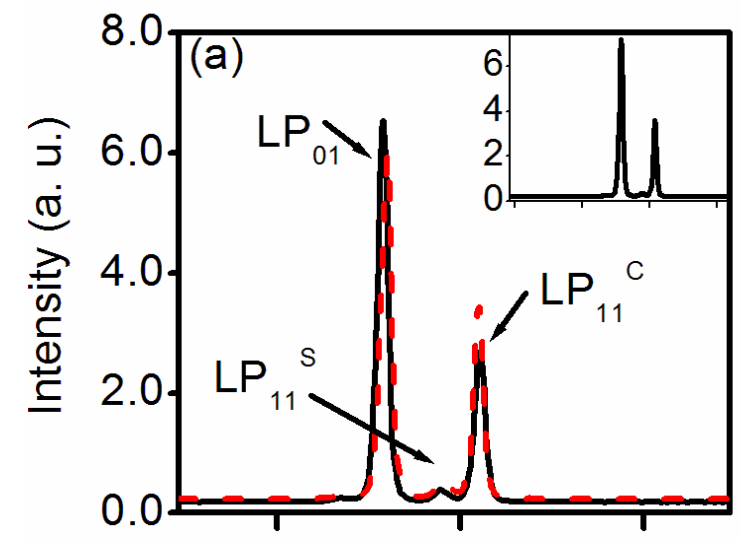

Frequency $(200 \mathrm{GHz} /$ div $)$

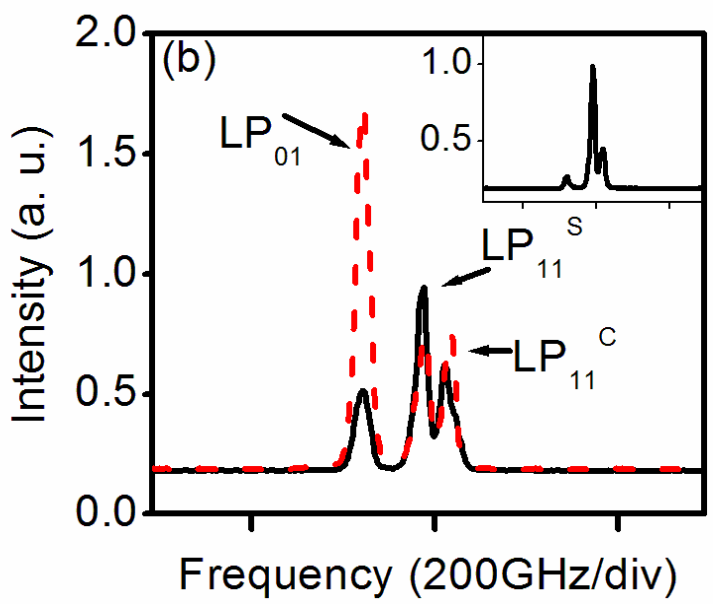

Fig. 4 


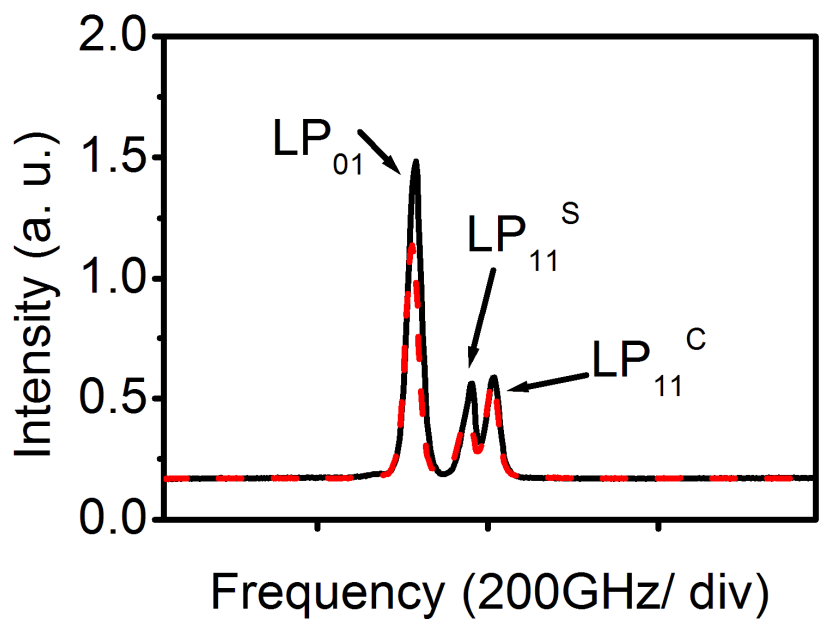

Fig. 5 

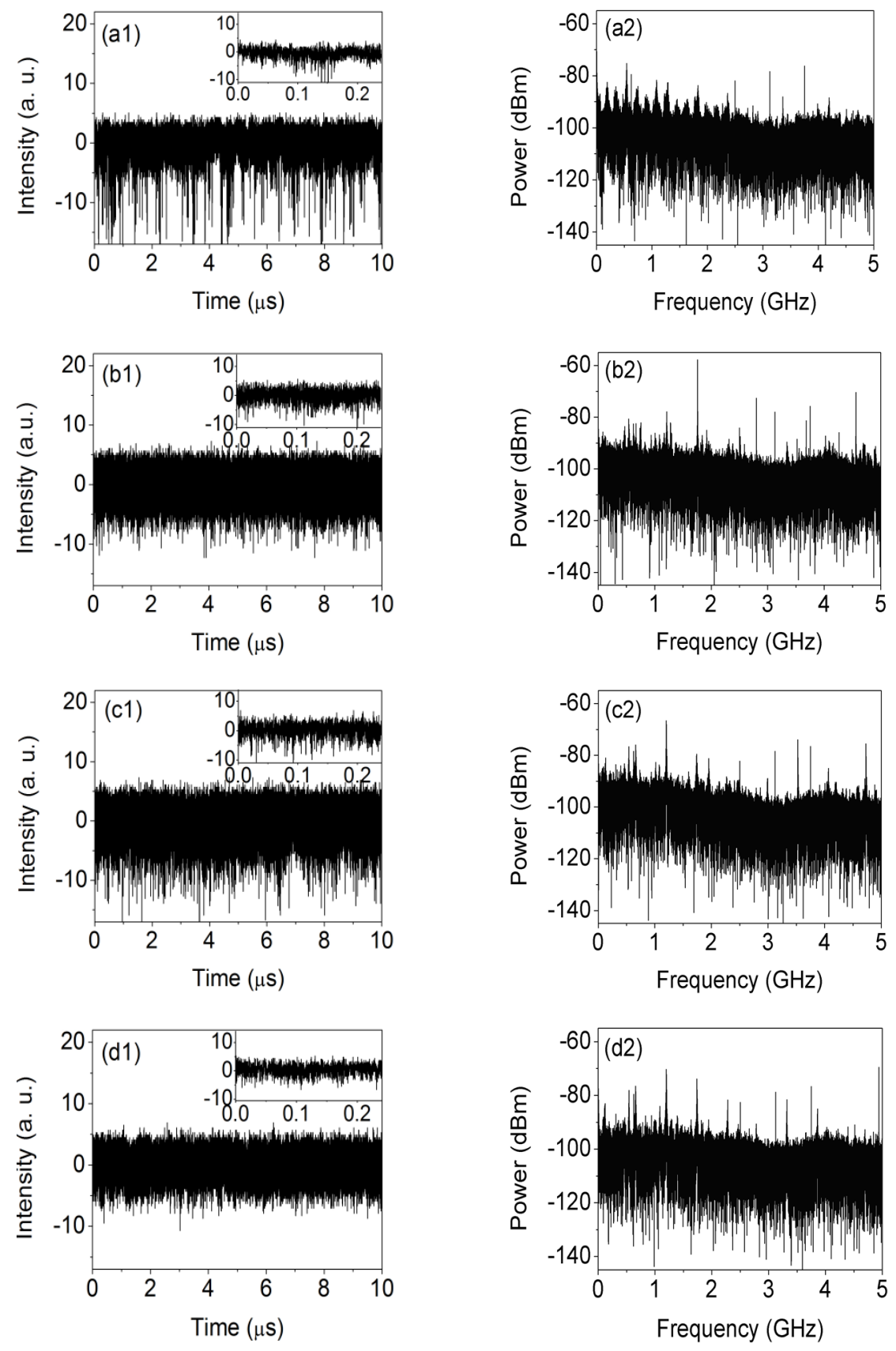

Fig. 6 

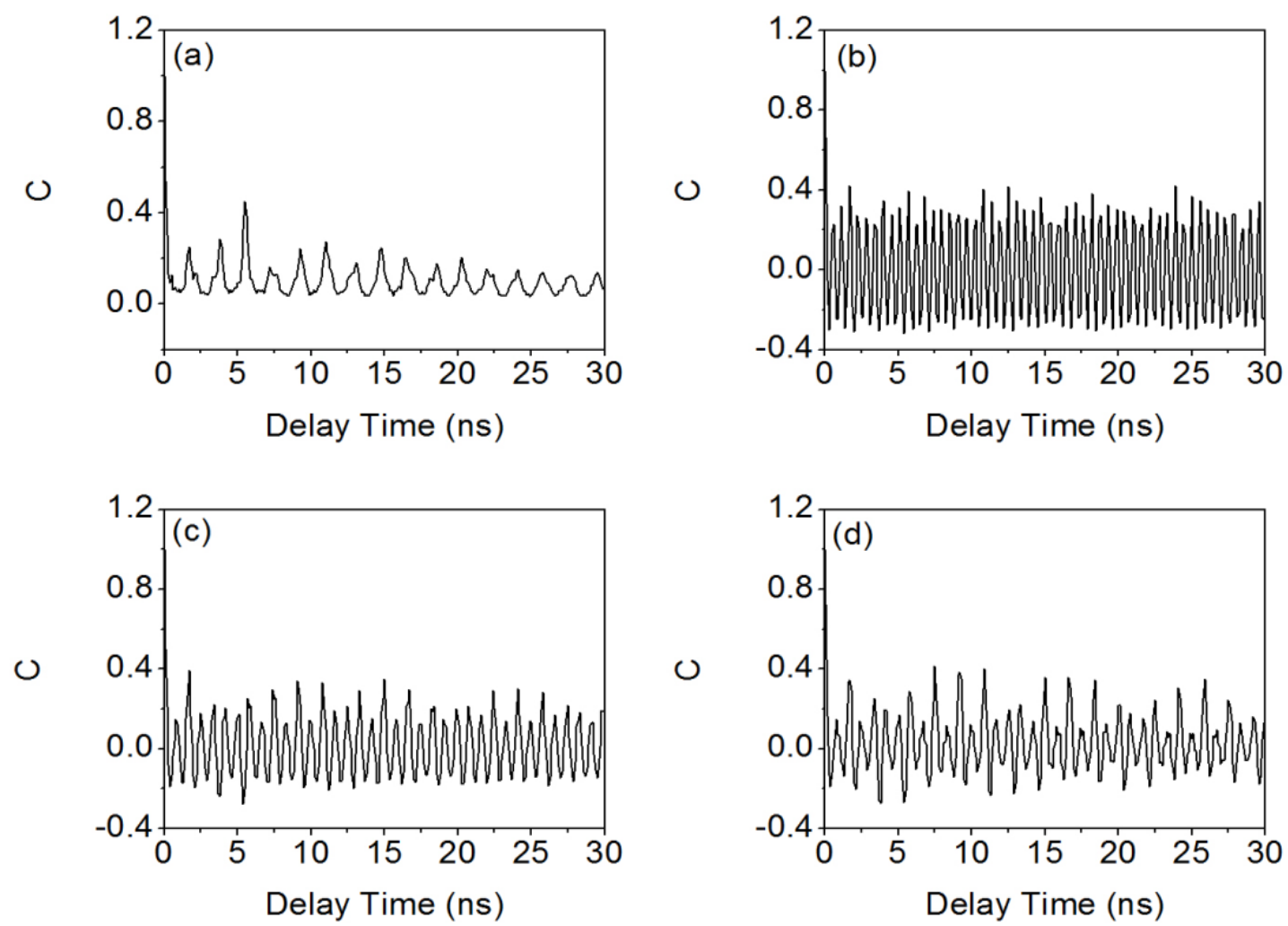

Fig. 7 

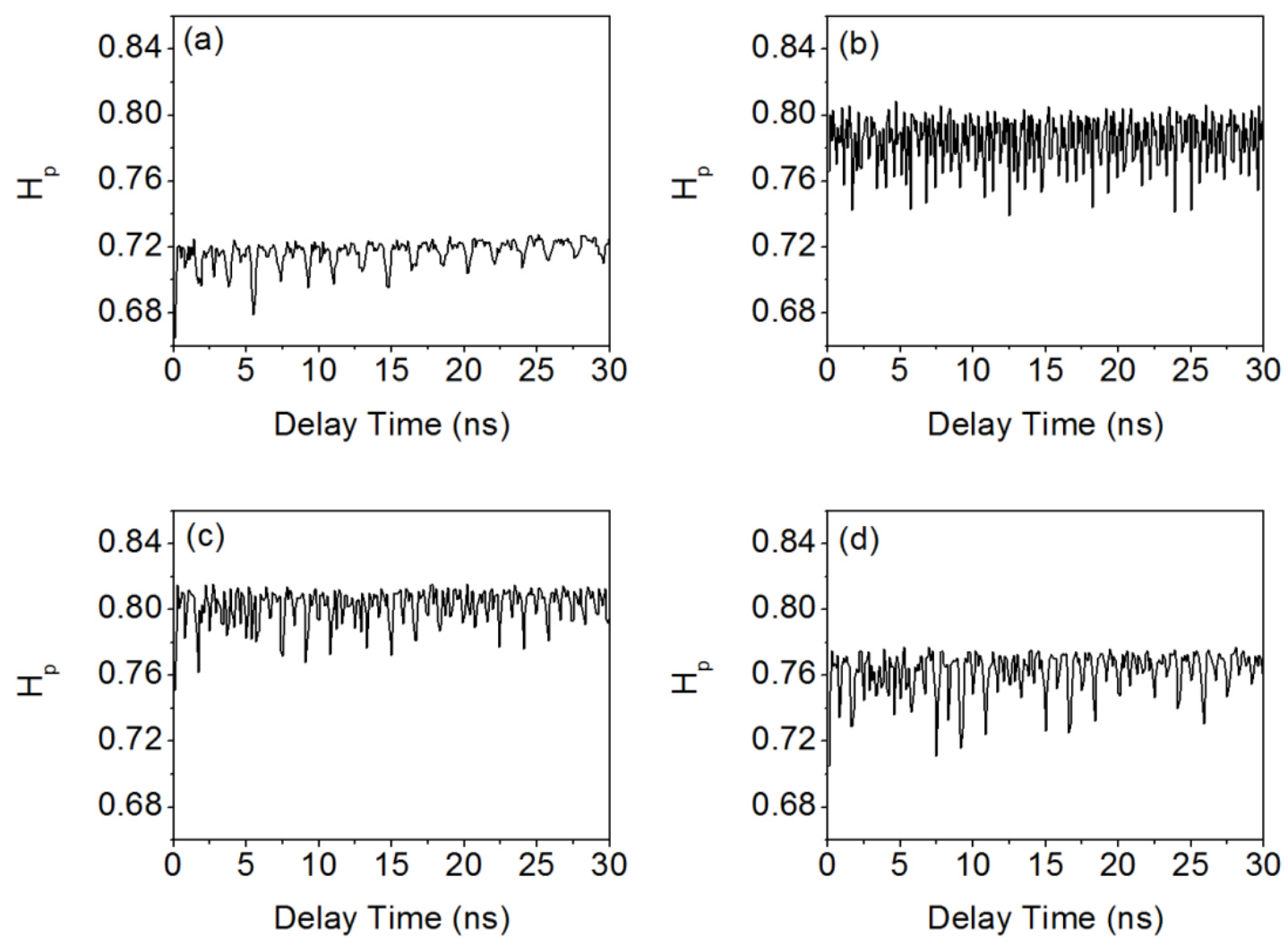

Fig. 8 

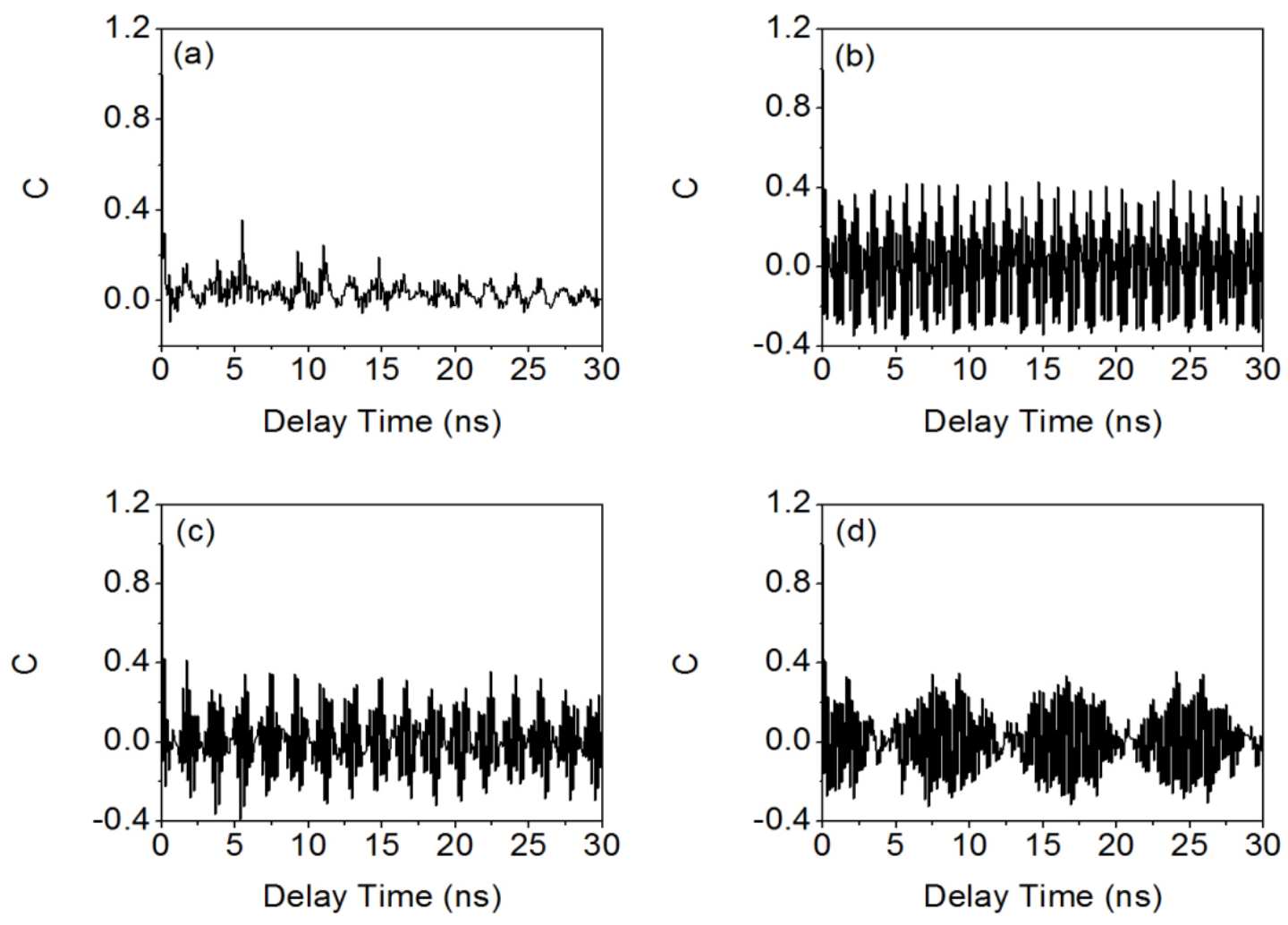

Fig. 9 

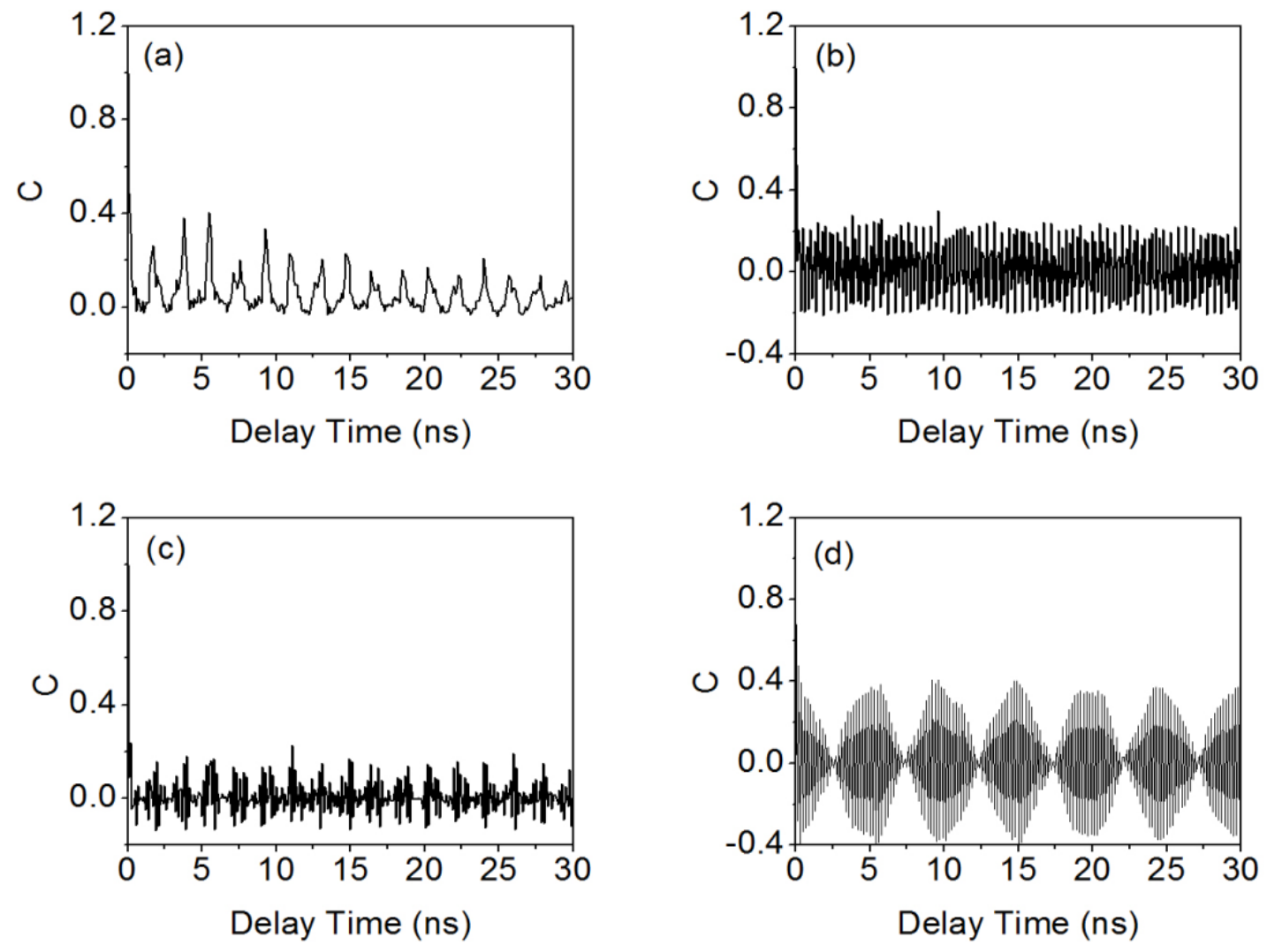

Fig. 10 

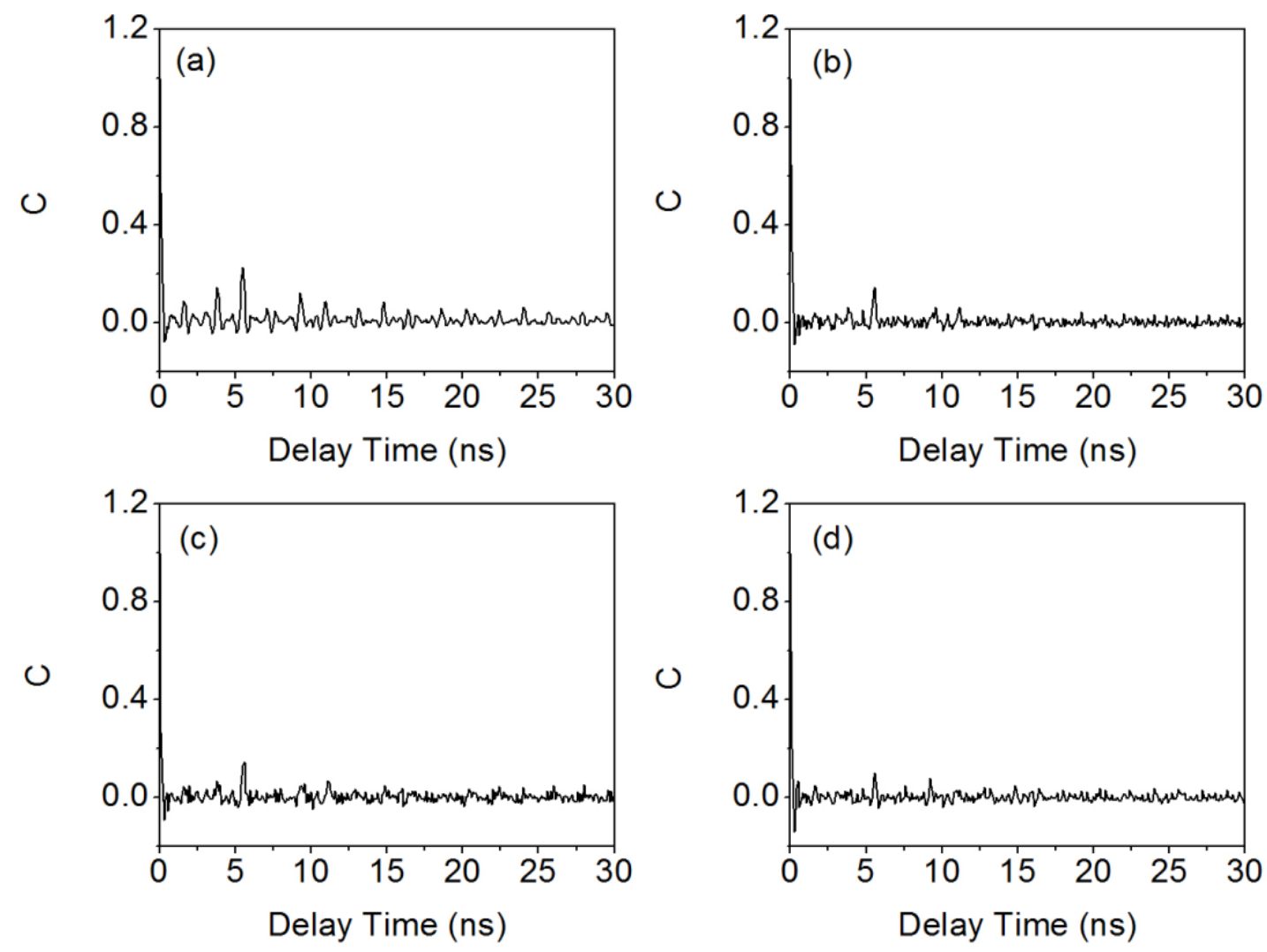

Fig. 11 

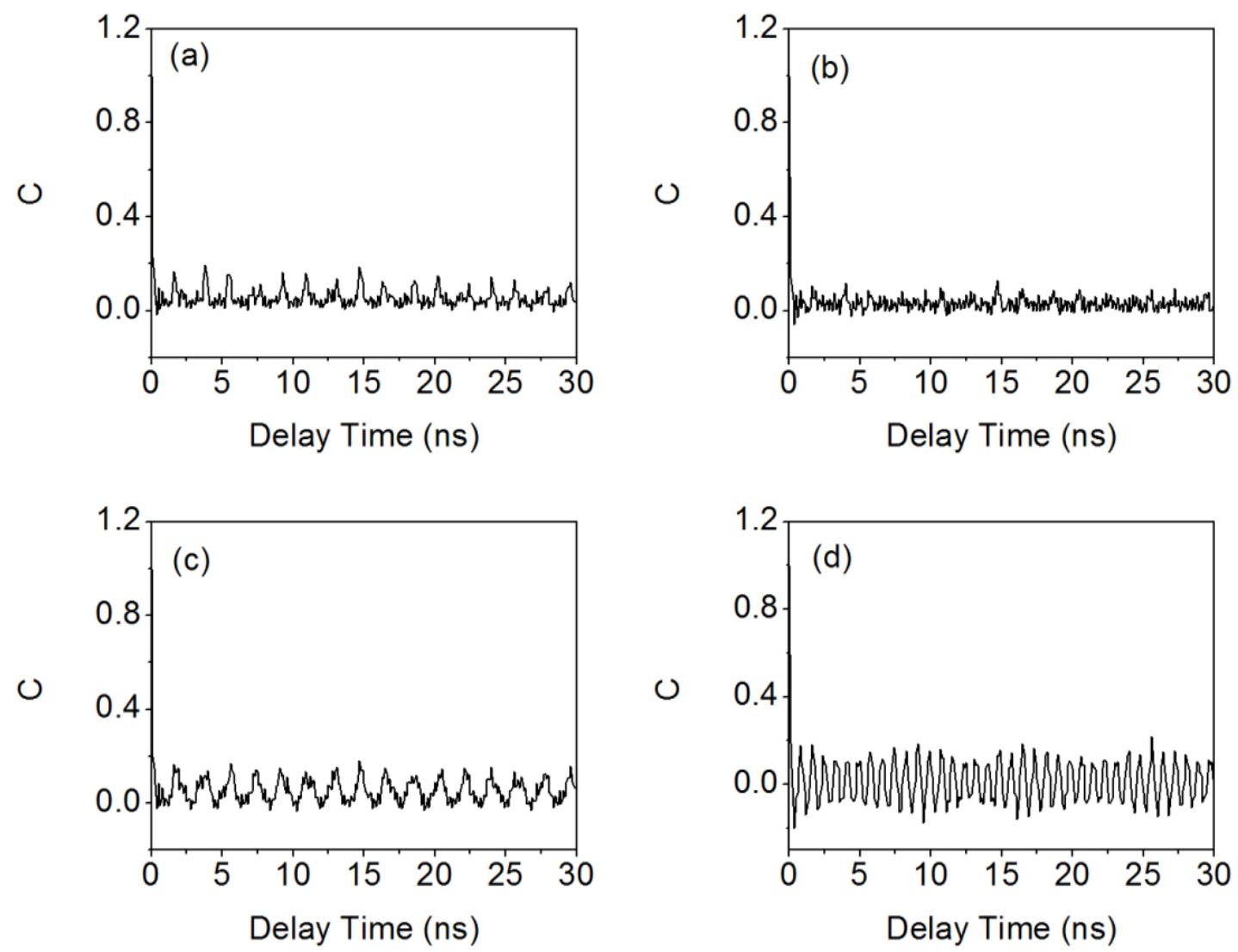

Fig. 12 

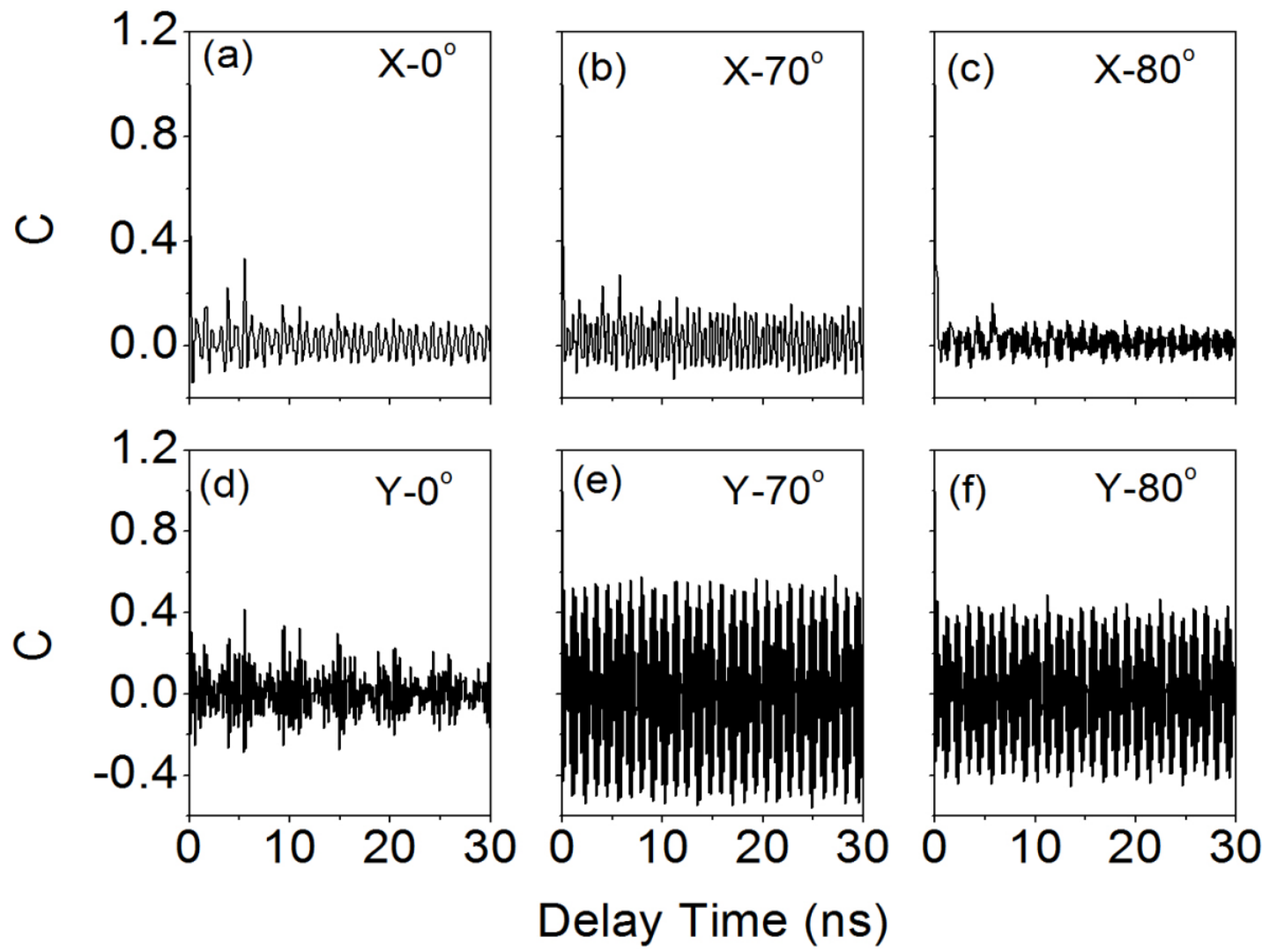

Fig. 13 

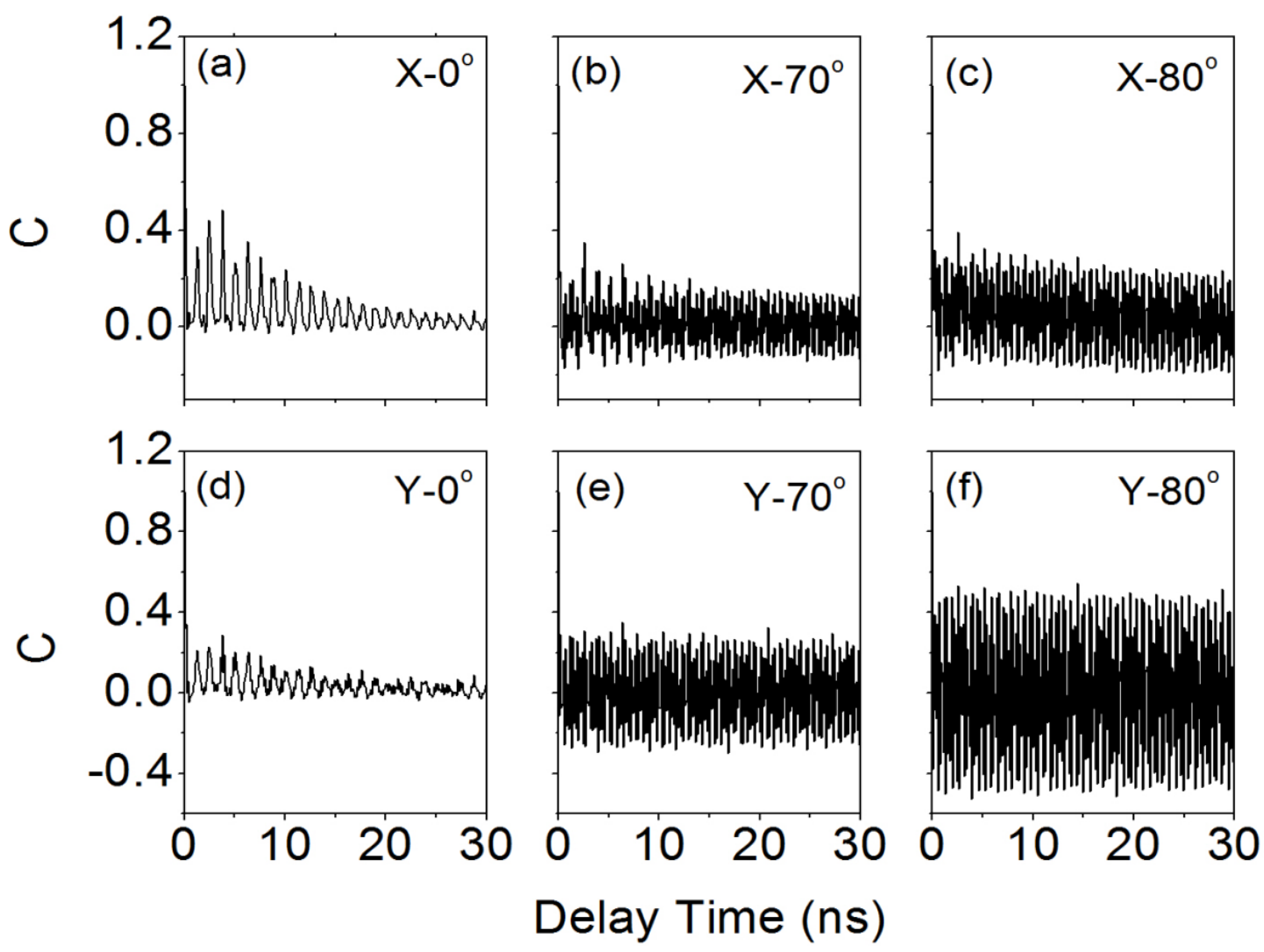

Fig. 14 


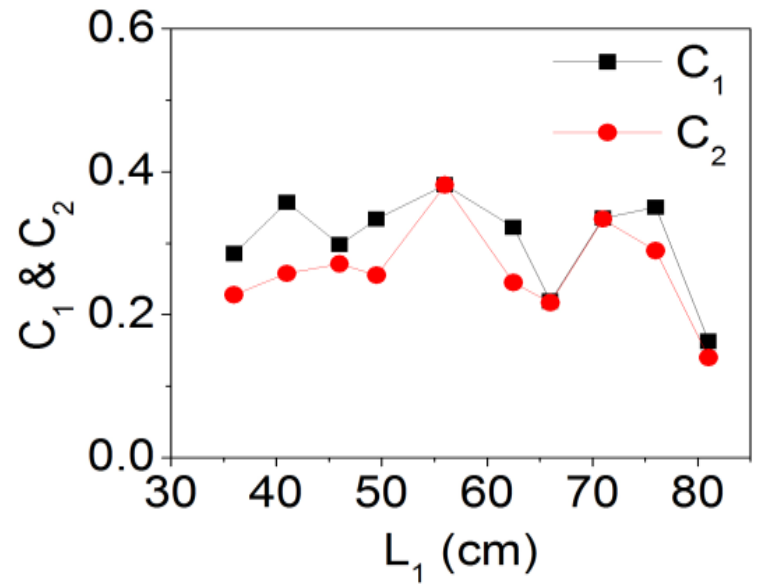

Fig. 15 